\title{
Isotope Channelomics of Chemoautotroph as the Predictor and Regulator of the Metal Deposit formation and the Weathering Factor
}

\author{
Gradov OV* \\ VL Tal'rose Institute for Energy Problems of Chemical Physics, Russian Academy of Sciences, Russia
}

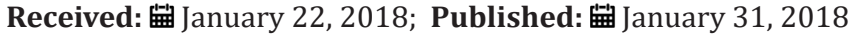

*Corresponding author: Gradov OV, VL Tal'rose Institute for Energy Problems of Chemical Physics, Russian Academy of Sciences, Russia 119334, Leninsky Prospect, 38/2, Moscow, Russia

\section{Editorial}

Chemoautotrophic and lithotrophic microorganisms are known to participate in the deposit formation and the rock weathering, but the results of such chemoautotroph-mediated biogeochemical activity and mass transfer strongly differ depending on the ionic composition of the medium, the salt conductivity effects and the Pourbaix diagrams of their medium, as well as on some other physico-chemical parameters which are often not considered as the active external factors for the sake of simplicity. The early biogeochemical considerations underlying the models and kinetic approaches to the analysis of such processes are mostly phenomenological and empirical and do not disclose the essence of the processes occurring at the boundary between the medium processed by the microorganisms and their active surface. Meanwhile, from the standpoint of biochemical physics, and especially biological kinetics, the mechanisms realizing at the interface or in its diffusion viscinity are decisive in such processes as the reagent input into the micro reactor-like biological compartments and aggregation upon biomineralization, which are usually biomembrane-mediated. Specificity of chemoautotrophic microorganisms to the chemically different media indicates the difference in their membrane properties within the natural mineral medium.
Any mechanisms determining the membrane activity in the inorganic medium are in fact the mechanisms of interaction between the biomembranes and the external medium, i.e. the mechanisms of the structural unit operation providing the transmembrane ion transport. Such structural units are represented by the cell ion channels or rather their complex known as channelome, providing the ion balance and the specificity of the membrane process kinetics. There are well known ion channels interacting with most of the chemical elements / agents which can be potentially involved in orogeny, mineralogenesis, metamorphism and the weathering processes as well as in the chemical taphonomy. The simplicity of the physico-chemical principles of designing the ion channel functional analogs and the early evolutional emergence of the primary ion channels allows to propose that at the very early stages (e.g. at the time of the origin of jaspillites) the channelomes of chemoautotrophic organisms could operate, including those of the extinct representatives of the 'shadow life'. The possibility of the isotope fractionation in microbial communities within the biogeochemical activity of the planetary microbiota ensures the participation of the channelome and membranome of the lithotrophs in the biological isotope fractionation during the deposit formation and weathering processes.
(C) Commons Attribution 4.0 License

To Submit Your Article Click Here:

Submit Article
DOI: $10.32474 /$ MAOPS.2018.01.000102

Citation: Gradov 0. Isotope Channelomics of Chemoautotroph as the Predictor and Regulator of the Metal Deposit formation and the Weathering Factor. Mod App Ocean \& Petr Sci 1(1)- 2018. MAOPS.MS.ID.000102. DOI: 10.32474/MAOPS.2018.01.000102.

(a) Factor. Mod App Ocean \& Petr Sci 1(1)- 2018. MAOPS.MS.ID.000102. DOI: 10.32474/MA0PS.2018.01.000102.
Modern Approaches in Oceanography and
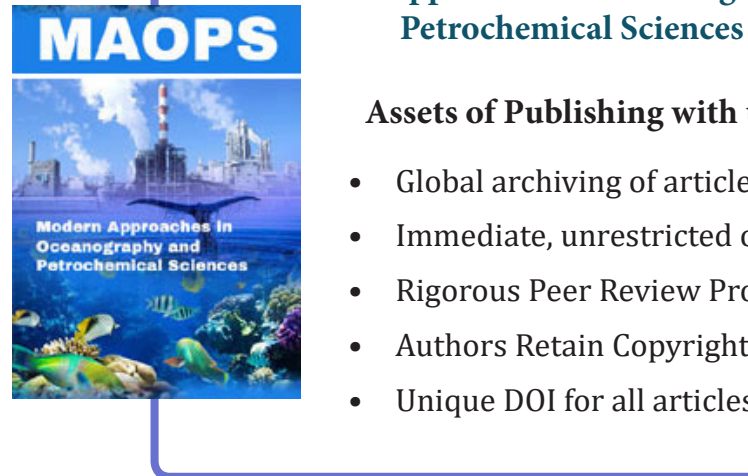

Assets of Publishing with us

- Global archiving of articles

- Immediate, unrestricted online access

- Rigorous Peer Review Process

- Authors Retain Copyrights

- Unique DOI for all articles 